\title{
具有鸭舵/尾舵多操纵面的导弹复合 控制方法研究
}

张明环 ${ }^{1}$ ，马万超 ${ }^{2}$, 郭云鹤 ${ }^{3}$, 徐胜利 ${ }^{3}$

1.西北工业大学 航天学院, 陕西 西安 710072; 2.上海航天技术研究院, 上海 201109;
3.上海机电工程研究所, 上海 201109

摘 要:提出了一种具有鸭舵/尾舵多操纵面的导弹复合控制方法。首先建立了导弹的数学模型并进 行了小扰动线性化,构建了鸭舵/尾舵复合控制系统,基于序列二次规划法设计了鸭舵/尾舵复合分配 策略,并在 Matlab/Simulink 平台上进行了仿真验证。仿真结果表明,设计的鸭舵/尾舵复合控制系统 不仅具有良好的动态性能和稳态性能,还能够在减小阻力的同时确保导弹从低空低速到高空高速都 具备高机动能力,证明了新算法具有较高的可信性和实用性。

\section{关 键 词: 导弹;鸭舵/尾舵;复合控制; 序列二次规划;仿真 \\ 中图分类号: V448.13 \\ 文献标志码 : A \\ 文章编号 : 1000-2758(2019)05-0962-06}

未来武器高空高速的发展趋势对防空系统提出 了更严峻的挑战,需要防空导弹从地面发射至 20 $30 \mathrm{~km}$ 的高空对目标进行拦截防御。但随着高度的 提升, 特别是 $20 \mathrm{~km}$ 以上, 空气变得非常稀薄, 导弹 的气动舵效将会大幅下降, 无法提供拦截所需的机 动过载能力, 从而导致防空导弹脱靶 ${ }^{[1-2]}$ 。

目前国内外针对该问题的解决方案集中于增加 直接控制力, 并通过直接力/气动力复合控制的方式 提升导弹的高空过载能力。文献 [3] 将气动力作为 主要的输人, 直接力作为辅助输人, 复合控制采用模 糊自适应的控制方法 ${ }^{[3]}$ 。文献 [4] 将复合控制系统 分为气动力和直接力 2 个子系统, 气动力子系统是 以过载跟踪的误差作为控制变量, 直接力子系统是 以过载跟踪误差及跟踪误差的变化率作为控制变 量 $^{[4]}$ 。文献 $[5]$ 采用了先进行气动力回路设计, 再 进行直接力回路设计的控制方案。气动力控制部分 采用滑模变结构控制方法, 直接力控制部分采用反 步法求解 ${ }^{[5]}$ 。研究成果表明, 导弹过载响应的上升 时间比单纯依靠气动力控制的响应时间下降一个数 量级, 能够在很大程度上提高导弹的控制系统的 性能。
但从另一方面来考虑, 气动力/直接力复合控制 的代价是要在导弹上增加新的能源。如此, 不仅增 加全弹重量, 挤压有效载荷空间, 还会极大地提升导 弹成本 ${ }^{[6-7]}$ 。为此, 本文提出了一种具有鸭舵/尾舵 多操纵面的导弹复合控制方法。通过增加一组鸭 舵,以提升导弹在高空飞行时的可用过载。该方案 的优点在于鸭舵的控制效率高, 铰链力矩小, 能降低 导弹跨声速飞行时过大的静稳定性, 因此适用于跨 空域、跨速域飞行的进攻或拦截武器。

本文拟基于导弹的小扰动线性化模型,构建鸭 舵/尾舵复合控制系统, 并采用序列二次规划法设计 鸭舵/尾舵的复合分配策略, 以保证导弹舵面效率最 大化, 从而满足跨空域、跨速域飞行的机动过载 需求。

\section{1 导弹数学模型}

\section{1 运动模型}

本文研究的导弹气动外形如图 1 所示, 导弹采 用轴对称外形,有 4 片鸭舵和 4 片尾舵。俯仰通道 和偏航通道采用鸭舵、尾舵联合控制, 滚转通道采用 
尾舵控制。由于俯仰通道与偏航通道完全对称, 因 此本文仅在纵向平面进行建模和设计工作。
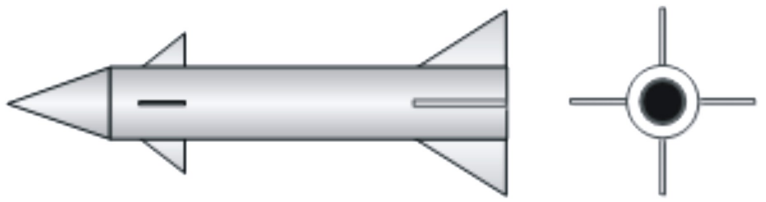

图 1 导弹气动外形

为简化弹体动力学模型, 把导弹视为刚体, 暂时 忽略地球自转的影响。导弹纵向平面的数学模型 如下：

$$
\left\{\begin{array}{l}
m \frac{\mathrm{d} V}{\mathrm{~d} t}=P \cos \alpha-X-m g \sin \theta \\
m V \frac{\mathrm{d} \theta}{\mathrm{d} t}=P \sin \alpha+Y-m g \cos \theta \\
J_{z} \frac{\mathrm{d} \omega_{z}}{\mathrm{~d} t}=M_{z} \\
\frac{\mathrm{d} x}{\mathrm{~d} t}=V \cos \theta \\
\frac{\mathrm{d} y}{\mathrm{~d} t}=V \sin \theta \\
\frac{\mathrm{d} \vartheta}{\mathrm{d} t}=\omega_{z}
\end{array}\right.
$$

式中, $\omega_{z}$ 为俯仰角速度, $M_{z}$ 为俯仰力矩, $J_{z}$ 为俯仰方 向的转动惯量, $x, y$ 为发射系坐标点, $V$ 为飞行速度, $\theta$ 为弹道倾角, $\vartheta$ 为俯仰角, $\alpha$ 为攻角。

\section{2 线性化模型}

对导弹数学模型进行小扰动线性化, 简化条 件为:

1) 采用系数冻结法, 即取弹道上某一时刻 $t$, 飞 行速度 $V$ 、高度 $H$ 、推力 $P$ 、质量 $m$ 、转动惯量 $J_{z}$ 均 不变;

2) 攻角 $\alpha$ 为小量, 即 $\sin \alpha \approx \alpha, \cos \alpha \approx 1$ 。

由此, 导弹动力学模型可线性化为

$$
\left\{\begin{array}{l}
\dot{\alpha}=\omega_{z}-\frac{q S C_{\mathrm{L}}^{\alpha}}{m V} \alpha-\frac{q S C_{\mathrm{L}}^{\delta_{T}}}{m V} \delta_{T}-\frac{q S C_{\mathrm{L}}^{\delta_{c}}}{m V} \delta_{c} \\
\dot{\omega}_{z}=\frac{M_{z}^{\omega_{z}}}{J_{z}} \omega_{z}+\frac{M_{z}^{\alpha}}{J_{z}} \alpha-\frac{M_{z}^{\delta_{T}}}{J_{z}} \delta_{T}+\frac{M_{z}^{\delta_{c}}}{J_{z}} \delta_{c}
\end{array}\right.
$$

式中, 导弹俯仰通道的空气动力阻尼系数 $a_{1}=$ $-M_{z}^{\omega_{z}} / J_{z}$, 导弹的静稳定动力系数 $a_{2}=-M_{z}^{\alpha} / J_{z}$, 导弹 的俯仰通道尾舵舵效率系数 $a_{3 \mathrm{~T}}=-M_{z}^{\delta_{T}} / J_{z}$, 导弹俯 仰通道的鸭舵舵效率系数 $a_{3 \mathrm{C}}=-M_{z}^{\delta_{c}} / J_{z}$, 俯仰通道 切线转动的角速度增量 $a_{4}=q S C_{\mathrm{L}}^{\alpha} / \mathrm{mV}$, 俯仰通道尾
舵舵面动力系数 $a_{5 \mathrm{~T}}=q S C_{\mathrm{L}}^{\delta_{T}} / m V$, 俯仰通道鸭舵舵面 动力系数 $a_{5 \mathrm{C}}=q S C_{\mathrm{L}}^{\delta_{c}} / m V_{\text {。 }}$

导弹纵向通道小扰动线性化模型为

$$
\left\{\begin{array}{l}
\Delta \ddot{\vartheta}+a_{1} \Delta \dot{\vartheta}+a_{2} \Delta \alpha=-a_{3 \mathrm{~T}} \Delta \delta_{\mathrm{T}}-a_{3 \mathrm{C}} \Delta \delta_{c} \\
\Delta \dot{\theta}-a_{4} \Delta \alpha=a_{5 \mathrm{~T}} \Delta \delta_{\mathrm{T}}+a_{5 \mathrm{C}} \Delta \delta_{c} \\
\Delta \vartheta=\Delta \theta+\Delta \alpha \\
\Delta n_{y}=\frac{V}{g} \Delta \dot{\theta}
\end{array}\right.
$$

选取尾舵偏角 $\delta_{\mathrm{T}}$ 和鸭舵偏角 $\delta_{c}$ 为输人, $\alpha$ 和 $\omega_{z}$ 为状态变量, $n_{y}$ 和 $\omega_{z}$ 为输出量, 可得导弹纵向状态 空间的简化形式

$$
\left\{\begin{array}{l}
{\left[\begin{array}{l}
\dot{\alpha} \\
\omega_{z}
\end{array}\right]=\left[\begin{array}{cc}
-a_{4} & 1 \\
-a_{2} & -a_{1}
\end{array}\right]\left[\begin{array}{c}
\alpha \\
\omega_{z}
\end{array}\right]+\left[\begin{array}{cc}
-a_{5 \mathrm{~T}} & -a_{5 \mathrm{C}} \\
-a_{3 \mathrm{~T}} & -a_{3 \mathrm{C}}
\end{array}\right]\left[\begin{array}{l}
\delta_{\mathrm{T}} \\
\delta_{\mathrm{C}}
\end{array}\right]} \\
{\left[\begin{array}{l}
n_{y} \\
\omega_{z}
\end{array}\right]=\left[\begin{array}{cc}
\frac{V a_{4}}{g} & 0 \\
0 & 1
\end{array}\right]\left[\begin{array}{c}
\alpha \\
\omega_{z}
\end{array}\right]+\left[\begin{array}{cc}
\frac{V a_{5 \mathrm{~T}}}{g} & \frac{V a_{5 \mathrm{C}}}{g} \\
0 & 0
\end{array}\right]\left[\begin{array}{l}
\delta_{\mathrm{T}} \\
\delta_{\mathrm{C}}
\end{array}\right]}
\end{array}\right.
$$

\section{2 鸭舵/尾舵复合控制系统}

导弹鸭舵/尾舵复合控制系统结构如图 2 所示:

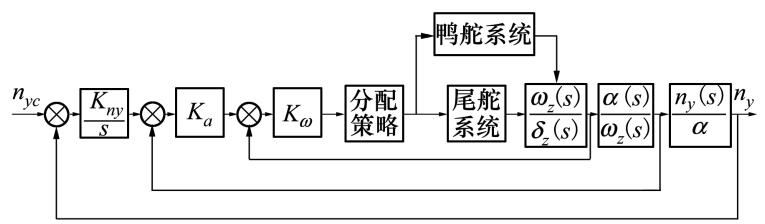

图 2 鸭舵/尾舵复合控制系统结构

导弹纵向通道传递函数为

$$
\frac{\omega_{z}(s)}{\delta_{z}(s)}=-\frac{a_{3}\left(s+a_{4}\right)}{s^{2}+\left(a_{1}+a_{4}\right) s+a_{2}+a_{1} a_{4}}
$$

式中, $a_{3}=a_{3 \mathrm{~T}}-a_{3 \mathrm{C}}$, 过载回路闭环传递函数为

$$
\frac{n_{y}(s)}{n_{y c}(s)}=\frac{B}{s^{3}+A_{1} s^{2}+A_{2} s+A_{3}}
$$

式中

$$
\begin{aligned}
& B=-a_{3} a_{4} K_{\omega} K_{\alpha} K_{n y} V / g \\
& A_{1}=a_{1}+a_{4}-K_{\omega} a_{3} \\
& A_{2}=a_{2}+a_{1} a_{4}-K_{\omega} a_{3} a_{4}-K_{\alpha} K_{\omega} a_{3} \\
& A_{3}=-a_{3} a_{4} K_{\omega} K_{\alpha} K_{n y} V / g
\end{aligned}
$$

采用极点配置方法求取反馈控制增益 $K_{\omega}, K_{\alpha}$ 和 
$K_{n y}$, 理想极点所对应的特征多项式为

$$
\operatorname{det}(s)=\left(T_{0} s+1\right)\left(s^{2}+2 \xi \omega_{d} s+\omega_{d}^{2}\right)
$$

式中, $\xi$ 和 $\omega_{d}$ 分别表征了理想极点所对应的阻尼比 和频率。

将(6)式和(7) 式中的系数对应相等, 可得控制 器参数设计关系式

$$
\left\{\begin{array}{l}
K_{\omega}=-\left(\frac{2 \xi \omega_{d} T_{0}+1}{T_{0}}-a_{1}-a_{4}\right) / a_{3} \\
K_{\alpha}=-\left(\frac{2 \xi \omega_{d}+T_{0} \omega_{d}^{2}}{T_{0}}-a_{1} a_{4}+a_{3} a_{4} K_{\omega}\right) / a_{3} K_{\omega} \\
K_{n y}=-\frac{\omega_{d}^{2} g}{T_{0} a_{3} a_{4} K_{\omega} K_{\alpha} V}
\end{array}\right.
$$

俯仰通道舵指令为

$$
\delta_{z c}=K_{\omega}\left\{K_{\alpha}\left[\frac{K_{n y}}{s}\left(n_{y c}-n_{y}\right)-\alpha\right]-\omega_{z}\right\}
$$

其分配策略为

$$
\left\{\begin{array}{l}
\delta_{z T}=K_{z 1} \delta_{z c} \\
\delta_{z C}=-K_{z 2} \delta_{z c} \\
K_{z 1}+K_{z 2}=2
\end{array}\right.
$$

本文采用序列二次规划法对鸭舵/尾舵分配系 数进行优化设计, 使导弹阻力最小化的同时尽可能 增大舵效,以减小导弹在高空的过载损耗

$$
\left\{\begin{array}{l}
\min f\left(K_{z 1}, K_{z 2}\right)=\left(\left|C_{\mathrm{D}}^{\delta_{\mathrm{T}}}\right|+\left|1 / m_{z}^{\delta_{\mathrm{T}}}\right|\right) K_{z 1}^{2}+ \\
\quad\left(\left|C_{\mathrm{D}}^{\delta_{z \mathrm{C}}}\right|+\left|1 / m_{z}^{\delta_{z \mathrm{C}}}\right|\right) K_{z 2}^{2} \\
K_{z 1}+K_{z 2}=2 \\
K_{z 1} \geqslant 0 \\
K_{z 2} \geqslant 0
\end{array}\right.
$$

\section{3 基于序列二次规划法的鸭舵/尾舵 分配策略}

一般非线性约束的数学规划问题是 ${ }^{[8-9]}$

$$
\begin{aligned}
& \min f(\boldsymbol{X}) \\
& \text { s.t. } g_{u}(\boldsymbol{X}) \leqslant 0 \quad(u=1,2, \cdots, p) \\
& h_{v}(\boldsymbol{X})=0 \quad(v=1,2, \cdots, m)
\end{aligned}
$$

式中, $\boldsymbol{X} \in \mathbf{R}^{n}$ 是决策变量, $f(\boldsymbol{X})$ 是目标函数, $g_{u}(\boldsymbol{X}), h_{v}(\boldsymbol{X})$ 分别是不等式约束函数和等式约束 函数。

利用泰勒展开把非线性约束问题 (11) 式的目
标函数在迭代点 $\boldsymbol{X}^{k}$ 简化成二次函数, 把约束函数简 化成线性函数后得到的就是如下的二次规划问题

$$
\begin{aligned}
& \min f(\boldsymbol{X})=\frac{1}{2}\left[\boldsymbol{X}-\boldsymbol{X}^{k}\right]^{\mathrm{T}} \nabla^{2} f\left(\boldsymbol{X}^{k}\right)\left[\boldsymbol{X}-\boldsymbol{X}^{k}\right]+ \\
& \quad \nabla f\left(\boldsymbol{X}^{k}\right)^{\mathrm{T}}\left[\boldsymbol{X}-\boldsymbol{X}^{k}\right] \\
& \text { s.t. } \nabla g_{u}\left(\boldsymbol{X}^{k}\right)^{\mathrm{T}}\left[\boldsymbol{X}-\boldsymbol{X}^{k}\right]+ \\
& g_{u}\left(\boldsymbol{X}^{k}\right) \leqslant 0(u=1,2, \cdots, p) \\
& \quad \nabla h_{v}\left(\boldsymbol{X}^{k}\right)^{\mathrm{T}}\left[\boldsymbol{X}-\boldsymbol{X}^{k}\right]+ \\
& h_{v}\left(\boldsymbol{X}^{k}\right)=0(v=1,2, \cdots, m)
\end{aligned}
$$

此问题是原约束最优化问题的近似问题,但其 解不一定是原问题的可行点。为此, 令 $\boldsymbol{S}=\boldsymbol{X}-\boldsymbol{X}^{k}$, 将上述二次规划问题变成关于变量 $\boldsymbol{S}$ 的问题, 即

$$
\begin{aligned}
& \min f(\boldsymbol{X})=\frac{1}{2} \boldsymbol{S}^{\mathrm{T}} \nabla^{2} f\left(\boldsymbol{X}^{k}\right) \boldsymbol{S}+\nabla f\left(\boldsymbol{X}^{k}\right){ }^{\mathrm{T}} \boldsymbol{S} \\
& \text { s.t. } \nabla g_{u}\left(\boldsymbol{X}^{k}\right){ }^{\mathrm{T}} \boldsymbol{S}+g_{u}\left(\boldsymbol{X}^{k}\right) \leqslant 0(u=1,2, \cdots, p) \\
& \quad \nabla h_{v}\left(\boldsymbol{X}^{k}\right)^{\mathrm{T}} \boldsymbol{S}+h_{v}\left(\boldsymbol{X}^{k}\right)=0(v=1,2, \cdots, m)
\end{aligned}
$$

令

$$
\left\{\begin{array}{l}
\boldsymbol{H}=\nabla^{2} f\left(\boldsymbol{X}^{k}\right) \\
\boldsymbol{C}=\nabla f\left(\boldsymbol{X}^{k}\right) \\
\boldsymbol{A}_{\text {eq }}=\left[\nabla h_{1}\left(\boldsymbol{X}^{k}\right), \nabla h_{2}\left(\boldsymbol{X}^{k}\right), \cdots, \nabla h_{m}\left(\boldsymbol{X}^{k}\right)\right]^{\mathrm{T}} \\
\boldsymbol{A}=\left[\nabla g_{1}\left(\boldsymbol{X}^{k}\right), \nabla g_{2}\left(\boldsymbol{X}^{k}\right), \cdots, \nabla g_{p}\left(\boldsymbol{X}^{k}\right)\right]^{\mathrm{T}} \\
\boldsymbol{B}_{\text {eq }}=\left[h_{1}\left(\boldsymbol{X}^{k}\right), h_{2}\left(\boldsymbol{X}^{k}\right), \cdots, h_{m}\left(\boldsymbol{X}^{k}\right)\right]^{\mathrm{T}} \\
\boldsymbol{B}=\left[g_{1}\left(\boldsymbol{X}^{k}\right), g_{2}\left(\boldsymbol{X}^{k}\right), \cdots, g_{p}\left(\boldsymbol{X}^{k}\right)\right]^{\mathrm{T}}
\end{array}\right.
$$

将(13)式变成二次规划问题的一般形式,即

$$
\begin{aligned}
& \min \frac{1}{2} \boldsymbol{S}^{\mathrm{T}} \boldsymbol{H} \boldsymbol{S}+\boldsymbol{C}^{\mathrm{T}} \boldsymbol{S} \\
& \text { s.t. } \boldsymbol{A} \boldsymbol{S} \leqslant \boldsymbol{B} \\
& \boldsymbol{A}_{\text {eq }} \boldsymbol{S}=\boldsymbol{B}_{\text {eq }}
\end{aligned}
$$

求解此二次规划问题,将其最优解 $\boldsymbol{S}^{*}$ 作为原 问题的下一个搜索方向 $\boldsymbol{S}^{k}$, 并在该方向上进行原约 束问题目标函数的约束一维搜索, 即可得到原约束 问题的一个近似解 $\boldsymbol{X}^{k+1}$, 反复迭代该过程, 直至求得 最优解。

针对 (10) 式所描述的二次规划问题, 在各参数 特征点 $(H, M a, \alpha)$, 采用序列二次规划法对分配系 数进行优化设计, 具体流程为:

1) 给定初值 $S_{0}=\left(K_{z 1}, K_{z 2}\right)$;

2) 求解 (10) 式所描述的二次规划子问题, 确 定搜索方向 $d_{k}$; 
$3)$ 令 $S_{k+1}=S_{k}+d_{k}$, 判断 $S_{k+1}$ 是否满足收玫条 件。若满足, 则取最优解为 $S^{*}=S_{k+1}$, 停止计算; 否 则返回 2) 继续迭代。

得到各参数特征点的最优解后, 利用增益调度 技术构建全飞行包线内的鸭舵/尾舵复合分配策略

$$
\left\{\begin{array}{l}
K_{z 1}=f_{1}(H, M a, \alpha) \\
K_{z 2}=f_{2}(H, M a, \alpha)
\end{array}\right.
$$

\section{4 仿真验证}

为验证本文所设计控制方法的有效性,构建以 下仿真场景: 导弹以 $70^{\circ}$ 俯仰角从地面发射, 加速爬 升至 $30 \mathrm{~km}$ 高度后定高巡航。仿真环境为 Matlab/ Simulink, 仿真步长为 $0.001 \mathrm{~s}$ 。仿真结果如图 $3 \sim 8$ 所示:

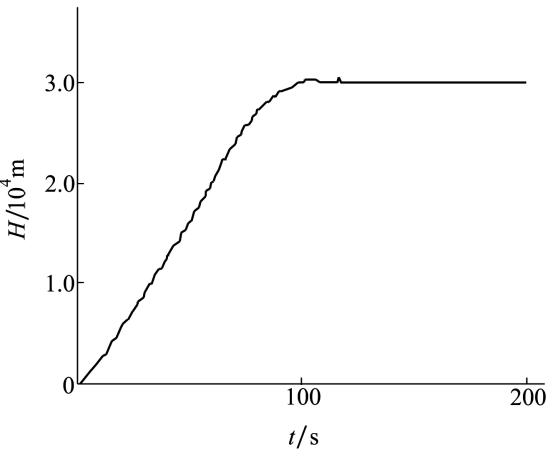

图 3 高度变化曲线

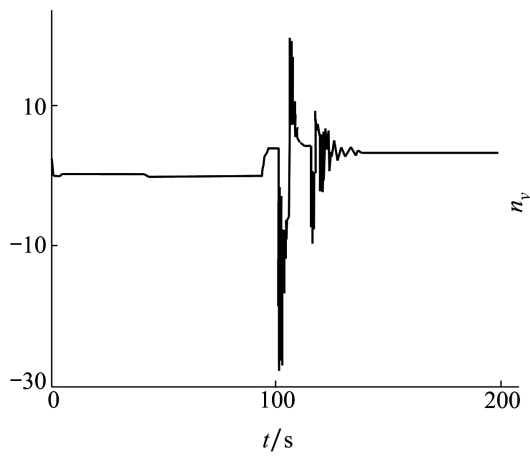

图 4 攻角变化曲线

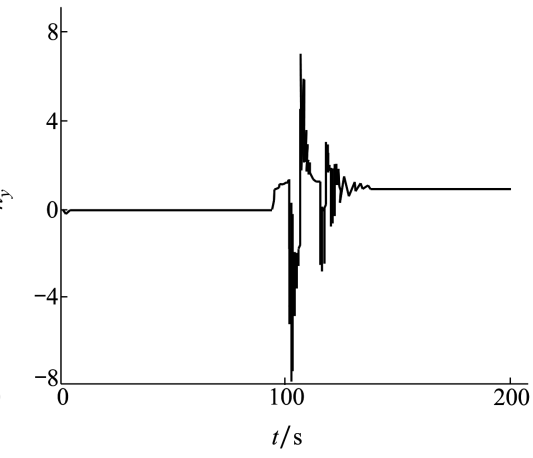

图 5 法向过载变化曲线
从图 3 可以看出, 导弹从地面发射后进行稳步 爬升, 在 $30 \mathrm{~km}$ 处能够迅速转平后进行定高巡航, 几 乎没有超调量。而从图 4 可以看出, 在高度转平时, 攻角仅进行了一次半的大幅抖动后便迅速衰减, 实 现飞行姿态的稳定控制。这些都证明了控制系统具 有良好的动态性能和稳态性能。

从图 5 可以看出, 在高度转平过程中, 鸭舵/尾 舵复合控制系统能够提供将近 $8 g$ 的瞬时过载, 证明 导弹即使在高空仍具备一定的机动能力。

从图 6 可以看出, 由于增加了一组鸭舵, 在实现 高空大过载机动时, 鸭舵/尾舵系统的满偏时间非常
短,并未导致控制系统发散。这证明增加鸭舵系统 能够有效解决导弹在高空飞行时气动舵效不足导致 的持续满偏问题。

从图 7 可以看出,在导弹低空飞行时,由于空气 密度较大, 尾舵舵效高, 因此规划的尾舵分配系数也 较大。飞行至 $15 \mathrm{~km}$ 左右时, 随着空气密度减小, 鸭 舵舵效逐渐升高至与尾舵舵效相近, 因此尾舵的分 配系数逐渐减小至 1 附近。随着高度的继续升高, 鸭舵舵效较大, 因此尾舵分配系数小于 1 。这证明 了本文序列二次规划的结果符合客观规律, 具有很 高的可信性。

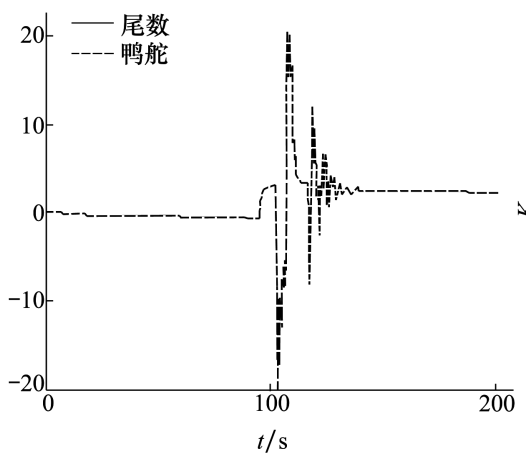

图 6 鸭舵/尾舵舵偏角变化曲线

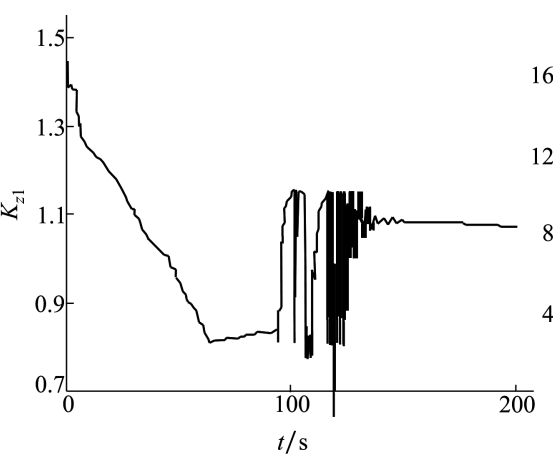

图 7 尾舵分配系数变化曲线

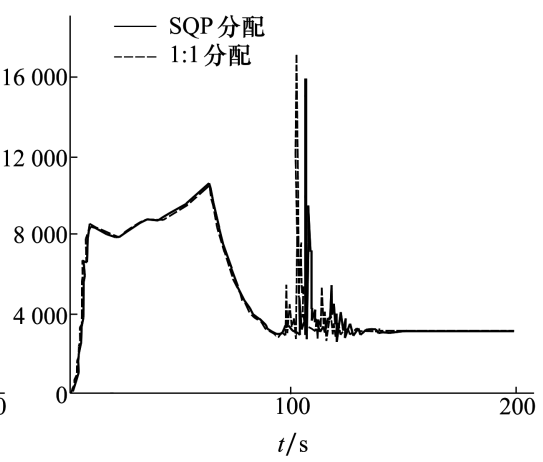

图 8 阻力变化曲线 
从图 8 可以看出,与工程上一般采用的 $1: 1$ 分 配方式相比, 本文采用序列二次规划法对舵指令进 行优化分配后, 能够有效减小导弹做机动过载时的 阻力。这证明了本文设计的分配策略非常有效。

\section{5 结 论}

本文提出了具有鸭舵/尾舵多操纵面的导弹复
合控制方法,并基于序列二次规划法设计了鸭舵/尾 舵复合分配策略。通过仿真验证, 证明了本文所设 计的复合控制系统不但具有良好的动态特性和稳态 特性, 还能够保证导弹从低空低速到高空高速都具 备高机动能力, 同时也能够有效减小导弹做机动过 载时的能量损耗。本文的研究为防空导弹的发展提 出了一个新的方向, 具有较高的应用价值和工程 意义。

\section{参考文献:}

[1] YUN Yuhang, TANG Shengjing, GUO Jie, et al. Robust Controller Design for Compound Control Missile with Fixed Bounded Convergence Time $[\mathrm{J}]$. Journal of Systems Engineering and Electronics, 2019, 29(1): 116-133

[2] CHAI Dong, FANG Yangwang, WU Youli, et al. Aerodynamic Force and Lateral Thrust Blended Control for Hypersonic Missile Using Fuzzy Control Algorithm[C] //34th Chinese Control Conference, 2015: 3567-3572

[3] WANG Zhengiie, LI Yangbo, LI Jing, et al. Modeling and Combined Control of Aerial Vehicle Based on Aerodynamic Force and Lateral Thrust [C] //The 2010 International Conference on Modelling, Identification and Control, 2010: 106-111

[4] SHI Z, MA W, ZHANG Y, et al. Lateral Thrust and Aerodynamics Compound Control System of Missile Based on Adaptive Fuzzy Control[C] //2012 Fifth International Symposium on Computational Intelligence and Design, 2012: 231-234

[5] 赵明元, 魏明英,何秋茹. 基于有限时间稳定和 Backstepping 的直接力/气动力复合控制方法 [ J ]. 宇航学报, 2010(9): 2157-2164

ZHAO Mingyuan, WEI Mingying, HE Qiuru. Research on Method of Lateral Jet and Aerodynamic Fins Compound Control Based on Finite Time Stability and Backstepping Approach[J]. Journal of Astronautics, 2010(9): 2157-2164 (in Chinese)

[6] 刘凯, 宋晓娜, 刘跃敏, 等. 导弹复合控制系统的自适应控制器设计 $[\mathrm{J}]$. 火力与指挥控制, 2018, 43(5): 878-881

LIU Kai, SONG Xiaona, LIU Yuemin, et al. Adaptive Control for Missile Compound Control Systems[J]. Fire Control \& Command Control, 2018, 43(5): 878-881 (in Chinese)

[7] 毕永涛, 王宇航, 姚郁. 直/气复合控制导弹的模型预测和自抗扰姿态控制设计 [ J ]. 宇航学报, 2015, 36(12) : 1373-1383

BI Yongtao, WANG Yuhang, YAO Yu. Attitude Control Design of Missiles with Dual Control Based on Model Predictive Control and Active Disturbance Rejection Control[J]. Journal of Astronautics, 2015, 36(12): 1373-1383 (in Chinese)

［8］易坚,陈勇,董新民,等. 多操纵面交叉耦合的 SQP 控制分配策略 $[\mathrm{J}]$. 系统工程与电子技术, 2016, 38(11): 2617-2623

YI Jian, CHEN Yong, DONG Xinmin, et al. SQP-Based Control Allocation for Multiple Effectors with Interactions [J]. Systems Engineering and Electronics, 2016, 38(11): 2617-2623 (in Chinese)

[9] 陈晓,王新民,周健. 基于序列二次规划法优化无人机飞行性能 [J]. 计算机仿真, 2012, 29(12): 99-102

CHEN Xiao, WANG Xinmin, ZHOU Jian. UAV Flight Performance Optimization Based on Sequential Quadratic Programming Method $[\mathrm{J}]$. Computer Simulation, 2012, 29(12) : 99-102 (in Chinese) 


\title{
A New Compound Control Method for Missile with Canard and Tail Rudders
}

\author{
ZHANG Minghuan ${ }^{1}$, MA Wanchao ${ }^{2}$, GUO Yunhe ${ }^{3}$, XU Shengli ${ }^{3}$ \\ $\left(\begin{array}{l}\text { 1.School of Astronautics, Northwestern Polytechnical University, Xi'an 710072, China; } \\ \text { 2.Shanghai Academy of Spaceflight Technology, Shanghai 201109, China; } \\ \text { 3.Shanghai Electro-Mechanical Engineering Institute, Shanghai 201109, China }\end{array}\right)$
}

Abstract : A new compound control method for missile with canard and tail rudders is presented in this paper. Firstly, the mathematical model of missile with canard/tail rudder is built and linearized using the small disturbance method. Then, the canard/tail rudder compound control system is created; the canard/tail rudder compound allocation strategy is designed based on the sequential quadratic programming method. Finally, the compound control method is verified on Matlab/Simulink. The simulation results show that the compound control method can not only ensure the high dynamic and steady state performance of the control system, but also ensure the high maneuverability of the missile in whole airspace with low drag, thus proving the algorithm in this paper to be reliable and effective.

Keywords : missile; canard/tail rudder; compound control; sequential quadratic programming; simulation; mathematical model; Matlab/Simulink 